\title{
Electrochemical Transistor Based on Tungsten Oxide with Optoelectronic Properties
}

\author{
Paul Grey ${ }^{1}$, Luís Pereira ${ }^{1}$, Sónia Pereira ${ }^{1}$, Pedro Barquinha ${ }^{1}$, Inês Cunha ${ }^{1}$, Rodrigo \\ Martins $^{1}$ and Elvira Fortunato ${ }^{1}$ \\ ${ }^{1}$ CENIMAT/i3N, Departamento de Ciência dos Materiais, Faculdade de Ciências e \\ Tecnologia, FCT, Universidade Nova de Lisboa and CEMOP-UNINOVA \\ Campus da Caparica \\ 2829-516 Caparica, Portugal \\ lmnp@fct.unl.pt,emf@fct.unl.pt
}

\begin{abstract}
This paper reports the integration of an electrochromic inorganic oxide semiconductor $\left(\mathrm{WO}_{3}\right)$ into an electrolyte gated transistor device. The resulting electrochromic transistor (EC-T) is a novel optoelectronic device, exhibiting simultaneous optical and electrical modulation. These devices show an On-Off ratio of $5 \times 10^{6}$ and a transconductance $\left(\mathrm{g}_{\mathrm{m}}\right)$ of $3.59 \mathrm{mS}$, for gate voltages $\left(\mathrm{V}_{\mathrm{G}}\right)$ between -2 and $2 \mathrm{~V}$, which, to the authors knowledge, are one of the best values ever reported for this type of electrochemical transistors. The simple and low-cost processing together with the electrical/optical performances, well supported into a comprehensive analysis of device physics, opens doors for a wide range of new applications in display technologies, biosensors, fuel cells or electrochemical logic circuits.
\end{abstract}

Keywords: tungsten oxides (VI); electrochromism; thin films; electrolyte-gated transistors; polymer electrolytes.

\section{Introduction}

Electrolyte-gated transistors (EGTs) and electrochemical transistors (ECTs) have received special attention over the last decade due to their advantages, mainly deriving from the use of solid electrolytes as gate dielectrics. These include low operating voltages, printability and solution processability, low contact resistances, the possibility to fabricate new device architectures, and high driving currents.[1] The understanding of the involved phenomena in electrolyte-gated transistor (EGT) operation is an important aspect to enable their integration in applications like biosensors, [2] microelectronics [3] or even the emergent area of textronics.[4]

EGTs using electrochromic (EC) semiconductors are particularly interesting as they combine electrical/ electrochemical effects with a simultaneous optical modulation.

The optical absorption of an EC material can be modified through double insertion of electrons and charge compensating ions, [5] which can be easily achieved by the gate field in an EGT structure. Being a reversible process, this gives an EC material the capability of transiting between two distinct oxidation states: colored and bleached. 
During the electrochemical reaction $\mathrm{WO}_{3}$ also undergoes a change in its electrical properties, namely in its conductivity, making it a candidate for implementation as the semiconductor layer in transistors, as initially demonstrated by Natan et al.[6] Furthermore, inorganic oxide semiconductors as $\mathrm{WO}_{3}$ possess higher chemical stability and higher conductance modulation than the organic semiconductors that compose most of the reported EGTs to date, which are great advantages for digital and analog circuit design with improved longevity.

The combination of both optoelectronic properties of EC-T opens possibilities in a wide range of applications and shows the value of the integration of smart materials into electronic circuitry.

\section{Relationship to Cyber-Physical Systems}

The use of electrolytes in active electronic components will be one of the fundamental building blocks of the interaction between common solid-state devices and ionic charge carriers. Figure 1 exemplifies the proposed interaction between an electrolyte and an ion permeable semiconductor.

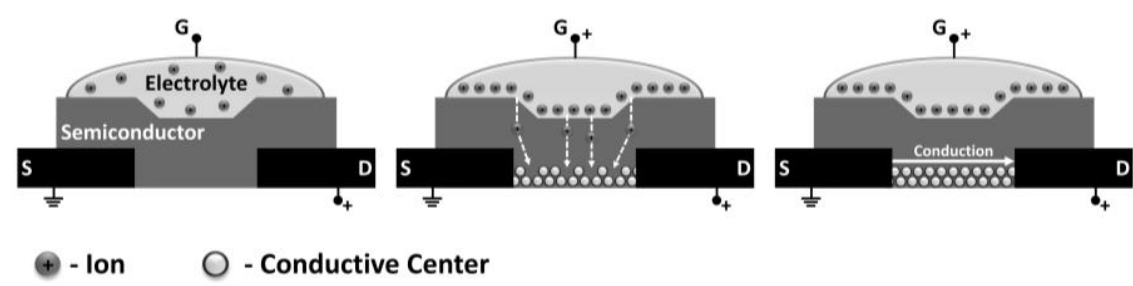

Fig. 1. Schematic representation of the interaction between an ion permeable semiconductor and an electrolyte in a transistor setup with source, drain and gate (S, D and G, respectively). In the present paper $\mathrm{WO}_{3}$ not only generates an electric response (conductivity change), but also an optical one (transmittance change), turning the system double redundant.

Electrolytes are a big part of modern society and naturally occur throughout the whole spectrum of science. For instance solely in physiological systems there exist a plethora of electrolytes, responsible for intra- and extracellular interactions as well as nerve and muscle function control, blood pressure and $\mathrm{pH}$. Consequently, reliable readout and double redundant values (electrical and optical) for electrolyte interpretation is crucial. Thus, creating a consistent interface between semiconductors and such electrolytes potentiates a main challenge for the development and study of EGTs. The technology of EGTs is therefore capable of adding gross value to a diverse range of cyber-physical systems (CPS), such as in chemical processes, healthcare and bio sensing schemes. 


\section{Results and Discussion}

The complete device structure is depicted in Figure 2. An interdigital source and drain titanium contact architecture was chosen in order to increase channel width $(3000$ $\mu \mathrm{m})$ and consequently the reached drain current of the devices. Furthermore an encapsulated device was fabricated, where the gate electrode (in this case an ITO coated PET foil) was extended over the electrolyte, resulting, not only, in a vertical architecture, but also in a self-passivation effect (Fig. 2 b and d).
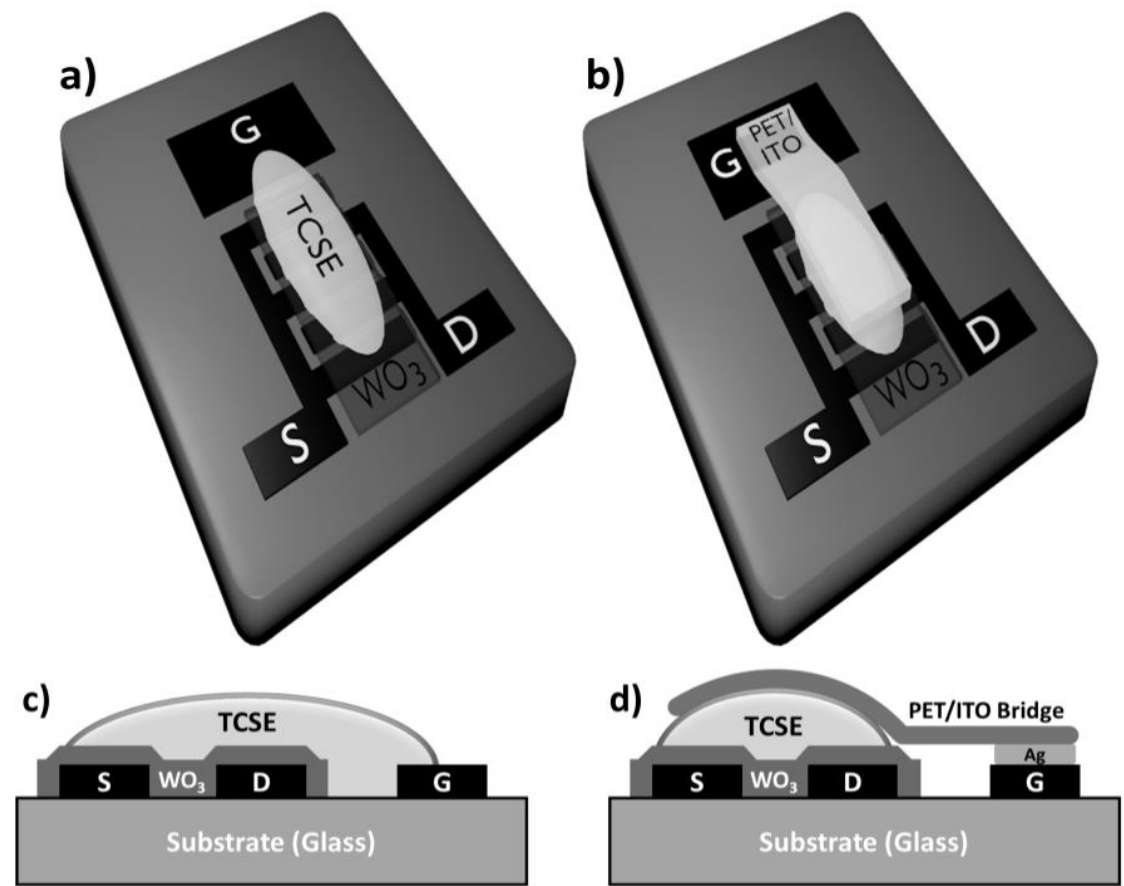

Fig. 2. Schematic device representation, where a) represents the planar and b) the vertical and encapsulated architecture and c) and d) show a longitudinal cross-section representation of both

of the device architectures. The PET/ITO foil is kept in place by capillary forces of the electrolyte and a silver glue $(\mathrm{Ag})$ on the gate electrode.

Subsequent characterization of the most crucial layers of the device was carried out. This includes electro-optical characterization of the sputtered $\mathrm{WO}_{3}$ thin-films $(100 \mathrm{~nm})$, Electrochemical impedance spectroscopy (EIS) of the drop-casted electrolyte (a thermosetting composite solid electrolyte - TCSE) and lastly electrical characterization of the final devices.

\subsection{Electro-Optical Measurements}

Electrochromism for $\mathrm{WO}_{3}$ is commonly introduced by reference to the following simplified redox reaction, also known as the double-injection model:[7] 


$$
W O_{3}(\text { transparent })+x e^{-}+x M^{+} \leftrightarrow M_{x} W O_{3}(\text { dark blue })
$$

where $\mathrm{M}^{+}$is an ion of the alkali metal group (e.g. $\mathrm{H}^{+}, \mathrm{Li}^{+}, \mathrm{Na}^{+}$or $\mathrm{K}^{+}$), provided by the electrolyte, $\mathrm{e}^{-}$represents electrons and parameter $x$, designated as the insertion coefficient, represents the proportion of electro-reduced tungsten sites. Following Equation 1, the pristine $\mathrm{WO}_{3}$ is transparent and the resulting tungsten bronze $\left(\mathrm{M}_{\mathrm{x}} \mathrm{WO}_{3}\right)$, for low $x$, exhibits its characteristic blue coloration due to photointervalence charge transfer (CT) between adjacent $\mathrm{W}^{\mathrm{V}}$ and $\mathrm{W}^{\mathrm{VI}}$ sites.[8]

It is widely accepted that the electro-optical centers are activated by the insertion of electrons and charge compensating ions, which distort and expand the lattice of the guest oxide, resulting in an overall modification of the optical properties and electronic structure of $\mathrm{WO}_{3}$. Charge carriers are trapped by $\mathrm{W}^{6+}$ octahedra, reducing them to $\mathrm{W}^{5+}$, while the alkali metals remain ionized in the interstitial space.[9] This results in a delocalization of electrons in the lattice and a charge carrier concentration increase of several orders of magnitude from the pristine oxide. This consequently increases the conductivity, which in terms of transistor operation is reflected in the transition between the Off- and On-states (oxidized and reduced states, respectively).

The $\mathrm{WO}_{3}$ film was deposited on an ITO coated glass substrate, which was submersed in a $\mathrm{Li}^{+}$rich liquid electrolyte in a conventional three-electrode setup. By applying a potential of 1 and $-1 \mathrm{~V}$ to the ITO film it is possible to influence the oxidation states (oxidized and reduced, respectively) and consequently monitor the change in transmittance at a specific wavelength $(\lambda=633 \mathrm{~nm})$, as well as the inserted charges into the $\mathrm{WO}_{3}$ film (Fig. 3). The wavelength corresponds to the transmittance of the characteristic blue coloration of reduced $\mathrm{WO}_{3}$ and it can be observed that a transmittance variation of over $90 \%$ is achieved. The inserted charges during reduction $(-1 \mathrm{~V})$ remain as free available charge carriers in the film and are extracted upon oxidation $(1 \mathrm{~V})$. A maximum of $26 \mathrm{mC}$ of charge induction was achieved, yielding (with a contact area of $0.833 \mathrm{~cm}^{2}$ ) a charge density of about $2 \times 10^{17} \mathrm{~cm}^{-2}$. However the process showed to be not completely reversible as charges might remain in the film after the reduction reaction (charge does not reach $0 \mathrm{mC}$ at $90 \mathrm{~s}$, see Fig. 3 ). Nonetheless sputtered $\mathrm{WO}_{3}$ films from previous works are capable of reaching very high lifetimes with more than 1000 cycles.[10] 


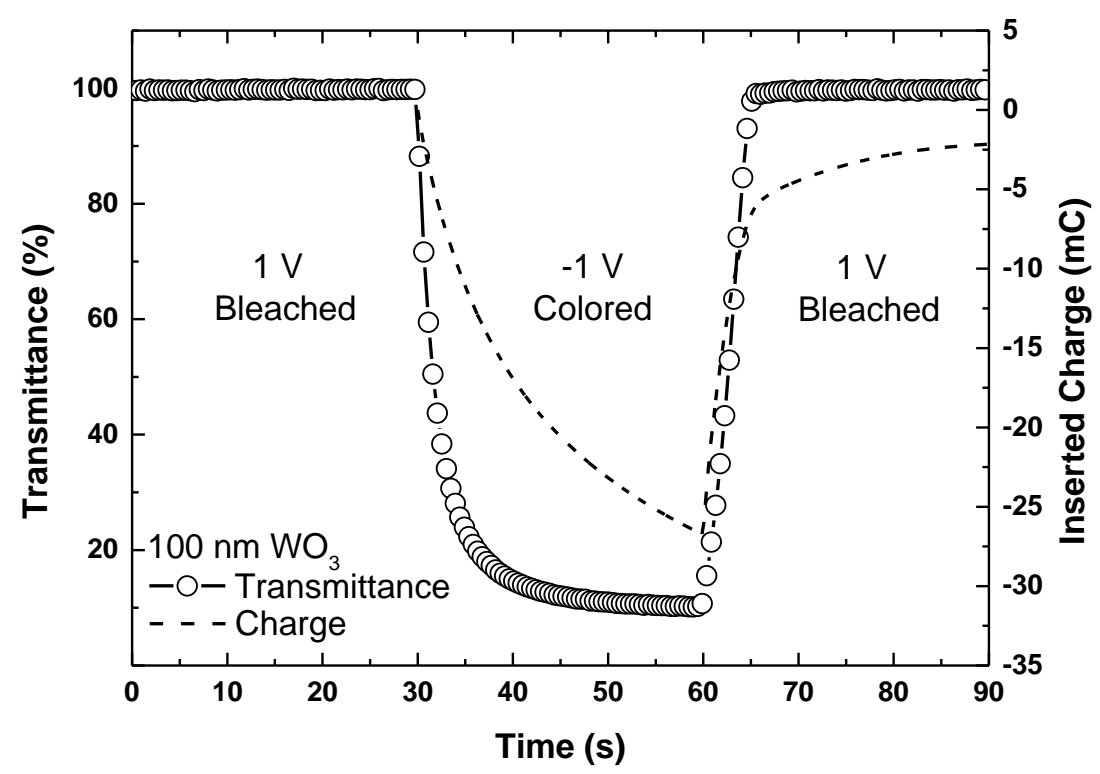

Fig. 3. Electro-optical measurements of a $100 \mathrm{~nm}$ thick $\mathrm{WO}^{3}$ film on ITO/Glass Substrate in a $\mathrm{Li}^{+}$rich electrolyte. Transmittance was measured during a full bleaching/coloration/bleaching cycle $\left(30 \mathrm{~s}\right.$ each) for the characteristic wavelength of reduced $\mathrm{WO}_{3}(633 \mathrm{~nm})$. The inserted and extracted available charge-carriers were also measured as a function of the duration of the applied potential.

This characterization technique proves the inherent optoelectronic behavior of $\mathrm{WO}_{3}$ and reveals its potential application in semiconducting devices, owing to reversible insulator-to-metal transitions, resulting from the electrochemical reaction.

\subsection{Electrochemical Impedance Spectroscopy (EIS)}

EIS is a well-known technique to determine the electrical properties of ionic materials, where an $a c$ potential at different frequencies (f) is applied to an electrochemical cell, so the total capacitor impedance $(Z)$ can be measured as a function of the frequency. This gives valuable information about ion migration, electric double layer (EDL) formation and its capacitance $\left(\mathrm{C}_{\mathrm{DL}}\right)$.

The electrochemical cell prepared for EIS is, as shown in Figure 4 a), composed of 3 distinct layers. Explicitly two ITO coated glass substrates and, sandwiched in between, the TCSE. The behavior and the obtained data of this cell can be described by an equivalent circuit model (ECM) for parameter determination (see Fig. 4 b).

This ECM (describing an ideally polarizable electrochemical cell), is composed of a resistance $\left(\mathrm{R}_{\mathrm{ext}}\right)$, associated to contact resistances (ITO), in series with a parallel $\mathrm{R}_{b} \mathrm{C}_{b}$ circuit and a constant phase element $(\mathrm{CPE})$. The $\mathrm{R}_{b} \mathrm{C}_{\mathrm{b}}$ circuit is associated to bulk properties of the electrolyte, namely the bulk resistance and capacitance, respectively. Whereas $R_{b}$ gives information about $\sigma_{i}, C_{b}$ accounts for the dipolar relaxation of electrolyte solvent molecules at higher frequencies. The CPE mimics a 
non-ideal capacitive behavior of the electric double layer, associated to interface inhomogeneities.[11]

Upon polarization of the electrochemical cell, ions migrate from one electrode to the other, causing charge built-ups and EDL formation. As can be observed in Figure 4 c) the ECM describes well the behavior of the cell throughout the whole frequency spectrum and serves for the determination of $\mathrm{C}_{\mathrm{DL}}$ and the ionic conductivity $\left(\sigma_{\mathrm{i}}\right)$.

Here, as we are dealing with an electrochemical transistor (ECT), $\sigma_{i}$ will be the parameter of interest. This parameter will determine how pronounced the effective net ion diffusion into the semiconductor will be and how fast the insulator-to-metal transition occurs. However, from an electrochemical point-of-view it is also important to determine the amount of accumulated charges at the interfaces $\left(\mathrm{C}_{\mathrm{DL}}\right)$, which, in terms of transistor characterization, accounts for charge induction (field-effect) into the semiconductor. It was shown that both effects (electrochemical reaction and fieldeffect) can contribute to the drain current.[12]

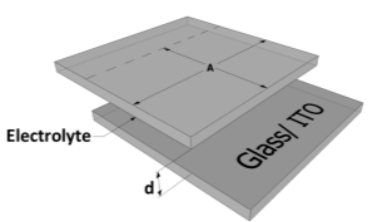

a)

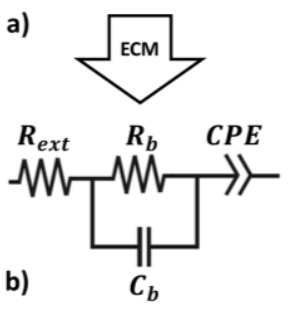

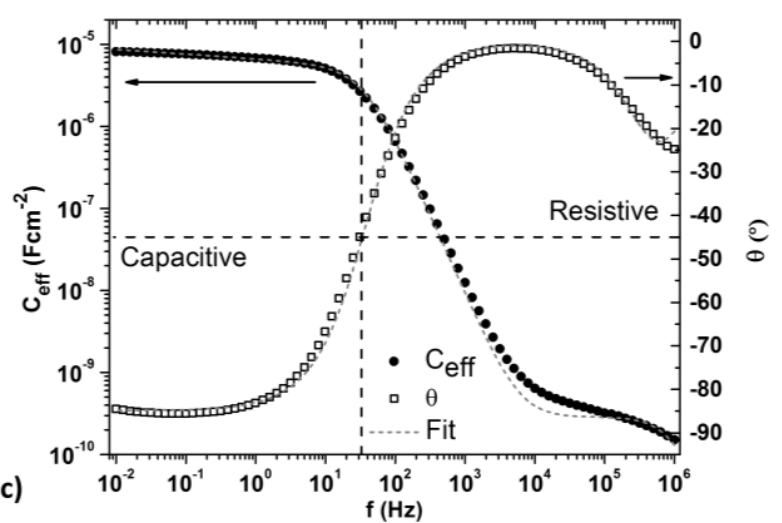

Fig. 4. Electrochemical Impedance Spectroscopy of the used Thermosetting Composite SolidElectrolyte (TCSE). Where a) shows the electrochemical cell (TCSE sandwiched in between two ITO (contact) coated glass substrates), b) represents the equivalent circuit model (ECM) used for the fittings and c) depicts the effective capacitance and the phase of the electrochemical cell in a frequency range between $10^{-2}$ and $10^{6} \mathrm{~Hz}$.

The ionic conductivity (for cell dimensions: $\mathrm{A}=5 \mathrm{~cm}^{2}$ and $\mathrm{d}=0.1 \mathrm{~mm}$ ) and $\mathrm{R}_{\mathrm{b}}=$ $87.34 \Omega$ from the fitting, $\sigma_{\mathrm{i}}$ of the electrolyte was determined as $2.29 \times 10^{-5} \mathrm{Scm}^{-1}$, using the standard resistivity equation. For $\mathrm{C}_{\mathrm{DL}}$ we considered the approach taken by Jović et al. [13] to empirically determine this value by using the fit for the CPE, yielding $\mathrm{C}_{\mathrm{DL}}=5.10 \mu \mathrm{Fcm}^{-2}$.

A clear advantage for this type of dielectric becomes evident as the achieved dielectric capacitance reaches very high values. However, as both characteristic parameters $\left(\sigma_{i}\right.$ and $\left.\mathrm{C}_{\mathrm{DL}}\right)$ are highly frequency-dependent, transistors working with these materials are often restricted to lower frequencies, where complete ionic 
relaxation can take place (capacitive regime in Fig. 4 c). Nonetheless, efforts are made towards an improvement of overall electrolyte behavior in order to augment the EGTs cut off frequency $\left(f_{c o}-\right.$ frequency where $\left.\mathrm{I}_{\mathrm{On}} / \mathrm{I}_{\mathrm{off}}=10\right)$. [14]

\subsection{Electrical Characterization}

Electrical characterization of the final devices (as depicted in Fig. 5 a) was conducted with a drain voltage $\left(\mathrm{V}_{\mathrm{D}}\right)$ as low as $1 \mathrm{~V}$ and a voltage sweep at the gate $\left(\mathrm{V}_{\mathrm{G}}\right)$ from -4 to $4 \mathrm{~V}$ and back at a scan-rate of $20 \mathrm{mVs}^{-1}$. Low-voltage operation of EGTs were found to be another strong factor of this type of devices, making their application in low power consuming applications feasible.
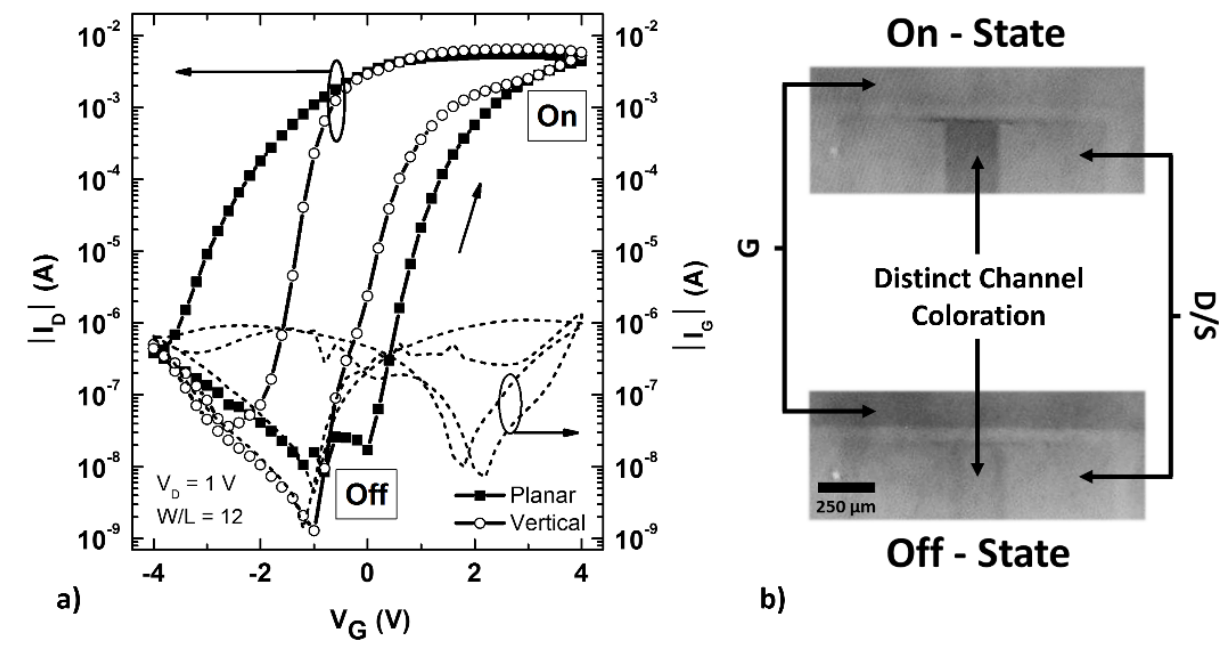

Fig. 5. a) Transfer characteristics of the fabricated planar and vertical EC-Ts and b) micrographs of channel region with distinct colorations between the On- and the Off-state. Curves obtained with $V_{D}=1 \mathrm{~V}$ and $V_{G}=(-4,4,-4) \mathrm{V}$ at a scan-rate of $20 \mathrm{mVs}^{-1}$. The arrow next to the main graphs indicates hysteresis direction.

The applied $V_{G}$ drives ion migration in the electrolyte (as schematized in Fig. 1) and promotes reversible electrochemical doping (reduction and oxidation) of the channel region. As can be observed in Figure 5 a, the fabricated EC-Ts start to switch close to $\mathrm{V}_{\mathrm{G}}=0 \mathrm{~V}$ to their respective On-states, remaining however with a normally On characteristic. As the channel reduces (and colors from transparent to blue, as seen in Fig. $5 \mathrm{~b}$ ) the drain current increases until reaching $\mathrm{mA}$ values around $\mathrm{V}_{\mathrm{G}}=2 \mathrm{~V}$, with leakage currents $\left(\mathrm{I}_{\mathrm{G}}\right)$ remaining in the order of $\mu \mathrm{A}$ (at most). The obtained OnOff ratios are considerable, reaching almost 7 orders of magnitude (vertical architecture). A hysteresis behavior is observed for both architectures planar and vertical, which is connected to the time-dependency of the electrochemical reaction, as well as ion migration processes. Nevertheless, it is possible to open and close both EC-Ts with symmetric VG (-4 and $4 \mathrm{~V}$ and -2 and $2 \mathrm{~V}$ for the planar and vertical architecture, respectively). 
Table 1 resumes crucial device parameters, evidencing an advantage for the vertical and encapsulated EC-T, reaching higher values in each aspect. Furthermore, the vertical EC-T showed higher lifetimes than the planar one due to the selfpassivation effect of the organic electrolyte, which is always subjected to environmental degradation factors.

Table 1. Crucial EC-T parameters, comparing the planar with the vertical structure. Values calculated from the curves of Figure 5a.

\begin{tabular}{ccccc} 
& $\mathbf{I o n}_{\text {On }} \mathbf{I o f f}_{\mathrm{of}}$ & $\mathbf{V}_{\mathbf{O n}}(\mathbf{V})$ & $\mathbf{S}_{\mathbf{s}}\left(\mathbf{V d e c}^{-1}\right)$ & $\mathbf{g}_{\mathbf{m}}(\mathbf{m S})$ \\
\hline \hline Planar & $6.55 \times 10^{5}$ & 0 & 0.28 & 2.24 \\
Vertical & $5.10 \times 10^{6}$ & -1 & 0.22 & 3.59 \\
\hline \hline
\end{tabular}

Analyzing Figure $5 \mathrm{a}$ and $\mathrm{b}$ together, the double functionality of the EC-Ts becomes visually clear, as it is possible to obtain a simultaneous electrical and optical response. The obtained information from these devices, regarding, for instance, biosensing applications, is on the one hand quantitative ( $\mathrm{I}_{\mathrm{D}}$ output) and on the other hand qualitative (color output). This leads to the above discussed double redundancy outputs, turning these devices highly reliable for sensing applications in healthcare or chemical and biological schemes. The results show how important but also how effective a controlled interaction between semiconductor/electrical components and ionic conductors is. A consistent integration of such devices into cyber-physical systems or as such will be feasible in the near future.

\section{Conclusion}

With this work we show an approach for a possible integration of ionic conductors (electrolytes) into electronic circuitry. The developed electrolyte-gated transistors (EGTs) based on the semiconductor $\mathrm{WO}_{3}$ exhibit double functionality (electrical and optical), as the electrochemical reaction of $\mathrm{WO}_{3}$ not only promotes a change in its coloration, but also a change of conductivity of several orders of magnitude. Characterization goes through electro-optical analysis of the $\mathrm{WO}_{3}$ film, electrochemical impedance spectroscopy of the electrolyte (a thermosetting composite solid-electrolyte - TCSE) and electrical characterization of the final devices. Two transistor architectures with identical $\mathrm{W} / \mathrm{L}=12$ were implemented (planar and vertical) in order to investigate structure influences and self-passivation effects. The best device (vertical) showed an On-Off ratio of almost 7 orders of magnitude (one of the highest ever reported for EGTs) for very low operation voltages $\left(\mathrm{V}_{\mathrm{D}}=1 \mathrm{~V}\right.$ and $\mathrm{V}_{\mathrm{G}}$ between -4 and $4 \mathrm{~V}$ ) and additional hysteresis reduction. In future works the dynamical switching of such devices will be in focus, as well as testing and optimizing different types of electrolytes. 
The presented research paves the way for sophisticated systems, where the analysis of electrolytes and the interaction of these with semiconductors is crucial. These devices could be integrated into applications as diverse as lab-on-chip, wearable electronics, healthcare monitoring and biosensors.

Acknowledgments. This work was supported by the FCT - Portuguese Foundation for Science and Technology, by the project EXCL/CTM-NAN/0201/2012 and by FEDER funds through the COMPETE 2020 Programme and National Funds through FCT under the project UID/CTM/50025/2013. The work was also supported by FP7 APPLE (grant number 262782-2) and SMART EC (grant number 258203) projects.

\section{References}

[1] Kim, S. H., Hong, K., Xie, W., Lee, K. H., Zhang, S., Lodge, T. P. ,Frisbie, C. D.: Electrolyte-Gated Transistors for Organic and Printed Electronics. Advanced Materials. 25, 1822 (2013)

[2] Berggren, M. ,Richter-Dahlfors, A.: Organic bioelectronics. Advanced Materials. 19, 3201-3213 (2007)

[3] Herlogsson, L., Crispin, X., Tierney, S. ,Berggren, M.: Polyelectrolyte-Gated Organic Complementary Circuits Operating at Low Power and Voltage. Advanced Materials. 23, 4684-+ (2011)

[4] Hamedi, M., Herlogsson, L., Crispin, X., Marcilla, R., Berggren, M. ,Inganas, O.: FiberEmbedded Electrolyte-Gated Field-Effect Transistors for e-Textiles. Advanced Materials. 21, 573-+ (2009)

[5] Granqvist, C. G.: Electrochromic tungsten oxide films: Review of progress 1993-1998. Solar Energy Materials and Solar Cells. 60, 201-262 (2000)

[6] Natan, M. J., Mallouk, T. E. ,Wrighton, M. S.: PH-SENSITIVE WO3-BASED MICROELECTROCHEMICAL TRANSISTORS. Journal of Physical Chemistry. 91, 648-654 (1987)

[7] Granqvist, C. G.: Progress in electrochromics: tungsten oxide revisited. Electrochimica Acta. 44, 3005-3015 (1999)

[8] Somani, P. R. ,Radhakrishnan, S.: Electrochromic materials and devices: present and future. Materials Chemistry and Physics. 77, 117-133 (2003)

[9] Azens, A., Hjelm, A., LeBellac, D., Granqvist, C. G., Barczynskab, J., Pentjuss, E., Gabrusenoks, J. ,Wills, J. M.: Electrochromism of W-oxide-based thin films: Recent advances. Solid State ionics. 86, (2007)

[10] Barquinha, P., Pereira, S., Pereira, L., Wojcik, P. J., Grey, P., Martins, R. ,Fortunato, E.: Flexible and Transparent WO3 Transistor with Electrical and Optical Modulation. Advanced Electronic Materials. 1, (2015)

[11] Barsoukov, E. ,Macdonald, J. R.: Impedance Spectroscopy Theory, Experiment, and Applications. John Wiley \& Sons, Inc., New Jersey (2005)

[12] Santos, L., Nunes, D., Calmeiro, T., Branquinho, R., Salgueiro, D., Barquinha, P., Pereira, L., Martins, R. ,Fortunato, E.: Solvothermal Synthesis of Gallium-Indium-Zinc- 
544 P. Grey, et al.

Oxide Nanoparticles for Electrolyte-Gated Transistors. Acs Applied Materials \& Interfaces. 7, 638-646 (2015)

[13] Jovic, V. D. ,Jovic, B. M.: EIS and differential capacitance measurements onto single crystal faces in different solutions - Part I: $\mathrm{Ag}(111)$ in $0.01 \mathrm{M} \mathrm{NaCl}$. Journal of Electroanalytical Chemistry. 541, 1-11 (2003)

[14] Liu, J., Herlogsson, L., Sawatdee, A., Favia, P., Sandberg, M., Crispin, X., Engquist, I. ,Berggren, M.: Vertical polyelectrolyte-gated organic field-effect transistors. Applied Physics Letters. 97, 3 (2010) 